\title{
Controlling docking, altitude and speed in a circular high-roofed tunnel thanks to the optic flow
}

\author{
Fabien Expert ${ }^{1}$ and Franck Ruffier ${ }^{1}$ \\ Aix Marseille University, CNRS, ISM UMR 7287, 13288, Marseille, France
}

\begin{abstract}
The new robot called BeeRotor we have developed is a tandem rotorcraft that mimicks optic flow-based behaviors previously observed in flies and bees. This tethered miniature robot $(\mathbf{8 0 g})$, which is autonomous in terms of its computational power requirements, is equipped with a 13.5-g quasi-panoramic visual system consisting of $\mathbf{4}$ individual visual motion sensors responding to the optic flow generated by photographs of natural scenes, thanks to the bio-inspired "time of travel" scheme. Based on recent findings on insects' sensing abilities and control strategies, the BeeRotor robot was designed to use optic flow to perform complex tasks such as ground and ceiling following while also automatically driving its forward speed on the basis of the ventral or dorsal optic flow. In addition, the BeeRotor robot can perform tricky manoeuvers such as automatic ceiling docking by simply regulating its dorsal or ventral optic flow in high-roofed tunnel depicting natural scenes. Although it was built as a proof of concept, the BeeRotor robot is one step further towards achieving a fully-autonomous micro-helicopter which is capable of navigating mainly on the basis of the optic flow.
\end{abstract}

\section{INTRODUCTION}

Unlike conventional aircraft, which are equipped with heavy, energy-consuming sensors, airborne insects such as flies and bees show remarkable prowess in terms of their autonomous navigation skills despite their small size. Several bio-inspired terrestrial and aerial robots have been developed during the past decade based on similar Optic Flow (OF) navigation principles [1]-[13]. These robots are mostly equipped with custom-made sensors performing optic flow computations based on analog Very-Large-Scale Integration (aVLSI) retinas [14], standard cameras [15] or even off-the-shelf mouse sensors [16]-[18]. Visual motion sensors, based on the "time of travel" scheme [19] inspired by elecrophysiological findings on the fly's Elementary Motion Detector neurons (EMDs) [20], have been implemented at our laboratory on both a terrestrial robot [1] and several tethered flying robots [21], [22]. Several visual motion sensors have been developed based on this scheme, and their performances

\footnotetext{
*This work was supported partly by CNRS Institutes (Life Science; Information Science; Engineering Science and Technology), the AixMarseille University, the French National Research Agency -ANR- (EVA project under ANR-ContInt grant number: ANR-08-CORD-007-04) and the European Commission via the CURVACE project. The CURVACE project acknowledges the financial support of the Future and Emerging Technologies (FET) programme within the Seventh Framework Programme for Research of the European Commission, under FET-Open grant number: 237940.

${ }^{1}$ F. Expert and F. Ruffier are with the Aix Marseille University, CNRS, ISM UMR 7287, 13288, Marseille, France (fabien.expert, franck.ruffier) d univ-amu.fr
}

have been assessed in terms of their resolution, accuracy, sensitivity, and invariance to contrast in real environments under a large range of illuminance conditions [23]-[26] and compared with those of off-the-shelf mouse sensors [27]. A few years ago, a biomimetic autopilot called OCTAVE (which stands for Optical altitude Control sysTem for Autonomous VEhicles) based on a ventral OF sensor was developed and integrated into a feedback loop driving a robot's lift to compensate for any deviations of the OF sensor's output from a given set-point [3], [22], [28], [29]. This simple autopilot enabled a miniature helicopter to perform challenging tasks such as takeoff, terrain following, reacting suitably to wind, and landing. Based on behavioral findings on bees [30], authors recently developed a visionbased autopilot called ALIS (which stands for AutopiLot using an Insect based vision System) [31] based on this concept of optic flow regulators [22], [28] and that of intertwined feedback loops [32]. The ALIS model showed how a "simulated flying insect" was able to travel along a 3-D tunnel by controlling both its speed and its clearance from the surrounding walls thanks to the dorsal, ventral and lateral optic flow sensing modalities.

Here we present a tethered lightweight (80-g) tandem rotorcraft called BeeRotor, which is equipped with a quasipanoramic visual system capable of assessing the ventral and dorsal optic flow in an indoor environment showing natural contrasts. Based on two feedback loops driving the robot's pitch angle and the lift, the embedded autopilot enables the BeeRotor robot to perform complex tasks such as ground and ceiling following, while automatically adjusting its forward speed so as to always maintain a safe distance from the ground and the ceiling and adapting its speed to the height of the high-roofed tunnel, as honeybees have been found to do [33], [34]. Based on behavioral studies on honeybees' grazing landings [35], authors started to use optic flow to enable wheeled robots [36], [37], rotorcrafts [10], [22] and fixed-wing Unmanned Aerial Vehicles (UAVs) [4], [38] to perform docking and landing manoeuvers. The BeeRotor robot can also perform tricky manoeuvers such as automatic ceiling docking by maintaining a constant OF with respect to the nearest wall, while reducing its forward speed.

In Section 2, the aeromechanical and electronical design of the BeeRotor robot and the 12-m long experimental environment in which the flying robot was tested are described. Section 3 focuses on the quasi-panoramic visual system and the method used to process the optic flow. In 


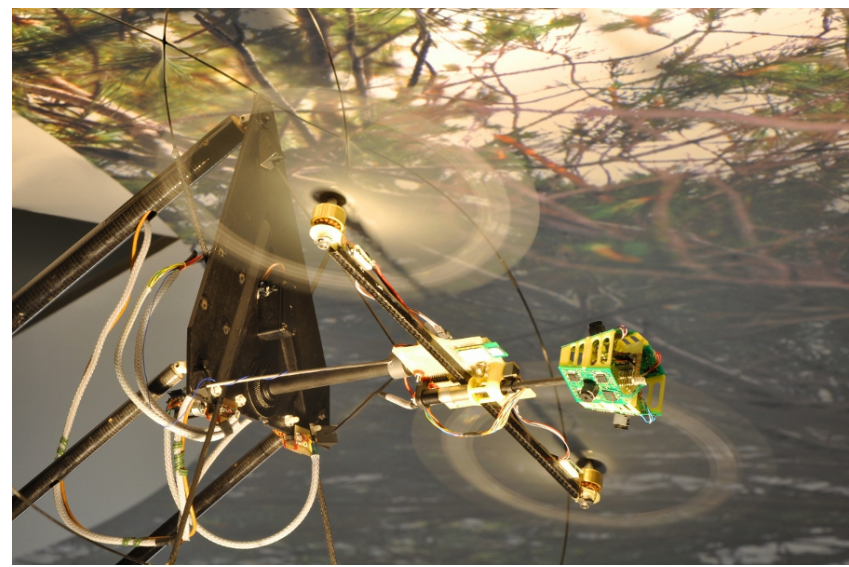

Fig. 1. Photograph of the $80 \mathrm{~g}$ BeeRotor robot in the experimental environment. Although the tandem rotorcraft was tethered to a pantographic arm for data monitoring and power supply purposes, it is autonomous in terms of its computational and signal processing power requirements. The two $20 \mathrm{~cm}$ propellers are driven by two 8.2-gram Brushless outrunner motors (Goldline LRK 13-4-15Y). A servomotor is used to orient the robot around its pitch axis, and a lightweight stepper motor is used to orient the eye, which is composed of 4 visual motion sensors sampling the visual environment with a 4 x 24-deg Field-Of-View. The robot is equipped with a full duplex serial bus via a Bluetooth module, for monitoring and communication purposes.

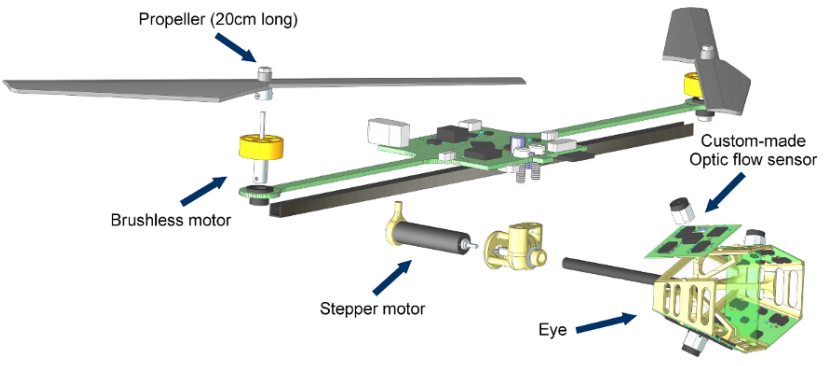

Fig. 2. Schematic CAD of the BeeRotor robot. It is composed of a main electronic board called the body, which carries most of the sensors and actuators, performs all the data processing and controls the rotorcraft. The pantographic arm to which this board is connected simply provides the aircraft with its power requirements. The main electronic board is composed of two elongated arms connected to two propellers controlled by Brushless outrunner motors. The robot's eye, which is set apart from the main board to prevent the visual field from being occluded by the propellers, is composed of 4 custom-made optic flow sensors based on an off-the-shelf photosensor array combined with the "time of travel" scheme. The eye can rotate independently from the body of the aircraft thanks to the presence of a stepper motor.

Section 4, the visuomotor control loops used to adjust the horizontal speed and the altitude of the BeeRotor robot are described. In section 5, the autonomous ground and ceiling following and automatic docking performances of the rotorcraft in the cluttered environment showing natural contrasts are presented.

\section{THE BEEROTOR ROBOT AND ITS ENVIRONMENT}

\section{A. Airframe}

The BeeRotor robot is a 80-gram tandem rotorcraft with a 47-cm wingspan, which is composed of a main electronic board weighing only 13 grams (called the "body") reinforced by a $25-\mathrm{cm}$ long carbon-fiber rod (Figures 1 and 2). It is propelled by two miniature 8.2-gram Brushless outrunner motors driving two $20-\mathrm{cm}$ long propellers borrowed from a X-UFO quadrotor. In this study, the pitch angle of the helicopter was driven by a servomotor placed on the pantographic arm but controlled by the robot itself. Although it is autonomous in terms of its computational requirements, the BeeRotor robot was connected to an external power supply via a 10-lead slip ring assembly at the extremity of the arm and also via a 40-lead slip ring assembly placed at the top of the central pole. The robot's eye, composed of 4 custommade visual motion sensors, can be rotated with respect to the body by a stepper motor coupled with a reductor, giving a resolution of $0.02^{\circ} /$ steps. The eye is placed $7 \mathrm{~cm}$ from the body to prevent the propellers from entering the visual field of the eye.

\section{B. Electronics}

The body of the rotorcraft is composed of a custom-designed electronic board, on which all the main sensors and actuators are set around a dsPIC microcontroller (33FJ128GP804). This microcontroller embeds the autopilot processing the data provided by the visual motion sensors and controls the actuators of the robot. This microcontroller communicates with the 4 visual motion sensors through a SPI bus and receives the outputs from the 201 -D Local Motion Sensors (LMS) computed at a rate of $1 \mathrm{kHz}$ (see Figure 4). The main electronic board is also equipped with:

- a tiny Bluetooth module (ALA from Free2move company) mediating information between the robot and a host computer,

- a custom-made positioning sensor, based on 2 tiny magnets with their orientation separated by an angle of $20 \mathrm{deg}$ and an A1391 Hall effect sensor, which determines the orientation of the eye with respect to the body.

\section{Environment}

The BeeRotor robot was traveling along a circular highroofed 12-m long tunnel, the floor and ceiling of which were covered with giant horizontal printed discs (inner diameter $2.4 \mathrm{~m}$, outer diameter $4.5 \mathrm{~m}$ ) depicting natural scenes (Fig. 3ab). The rotorcraft was tethered to the end of a light, counterbalanced whirling pantographic arm driven in elevation and azimuth by the rotorcraft's main force, which provided the lift and forward propulsive force. The pantographic arm was equipped on the elevation axis with a servo-potentiometer giving the robot's altitude, and on the travel axis, with an optical encoder giving the robot's azimuth angle, and hence the horizontal distance travelled and the robot's forward speed. These data are collected on the host computer using 
a)
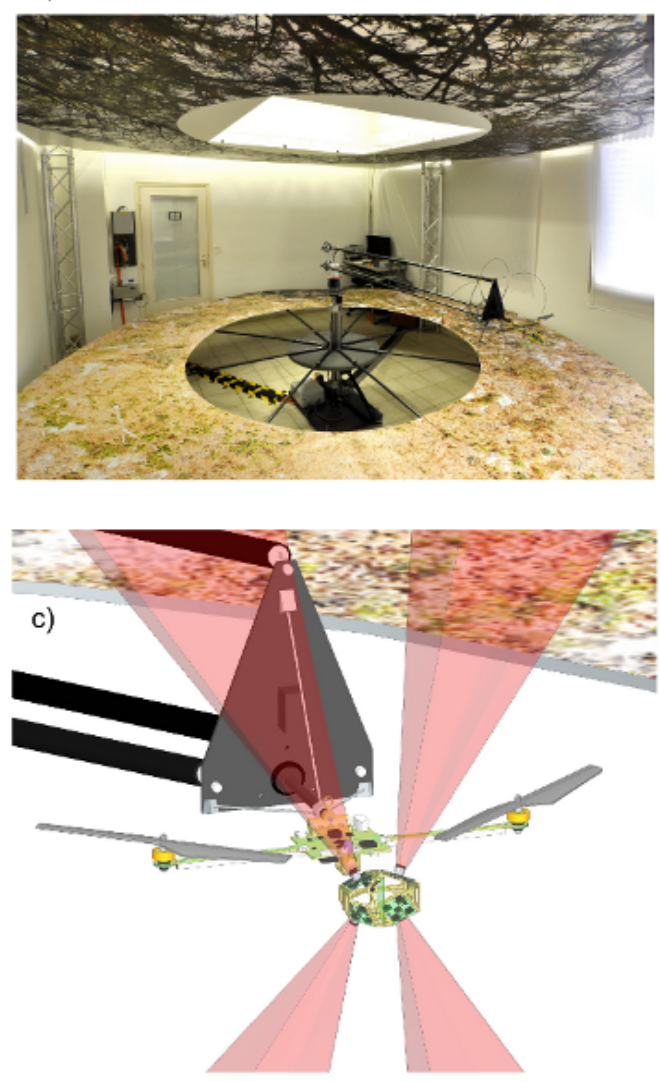

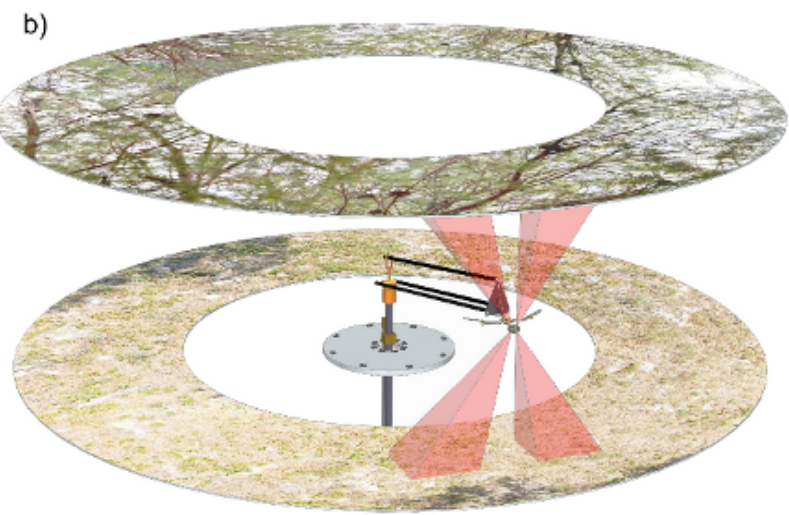

d)

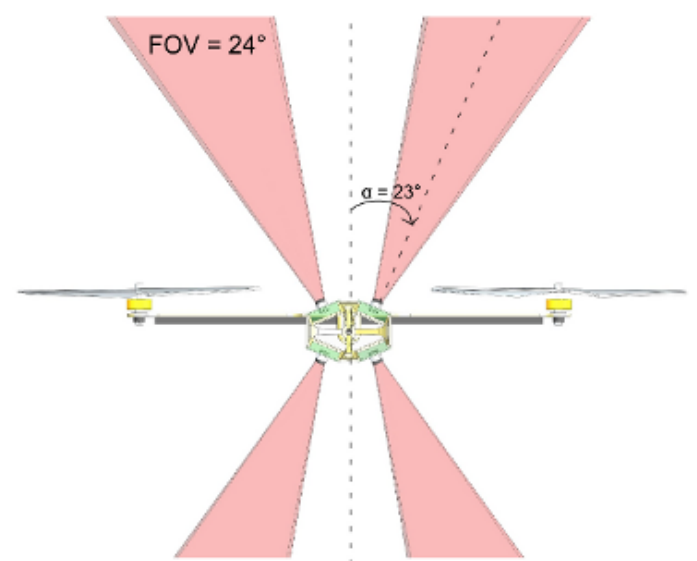

Fig. 3. a) Photograph of the experimental setup, where the BeeRotor robot is placed in the visual environment created for this purpose. The robot was made to travel along a 12-m long high-roofed tunnel, the floor and ceiling of which were covered with photographs depicting natural scenes. The rotorcraft was tethered to the end of a light, counterbalanced whirling pantographic arm, which was driven in elevation and azimuth by the aircraft's lift and propulsive forces. b) CAD drawing of the aircraft with the patterned ceiling and ground. The Fields Of View of the 4 visual motion sensors indicated here show that the aircraft was looking only at the printed discs covered by natural scenes. c) Magnified view of the aircraft's CAD. The distance between the eye and the body prevented the propellers from occluding the visual field of the 4 visual motion sensors during the robot's flight. d) The robot's 4 visual motion sensors were looking Up-Forward, Up-Backward, Down-Forward and Down-Backward with a FOV of $24^{\circ}$. The main direction of each visual motion sensor was tilted around the robot's pitch axis at an angle of $\alpha= \pm 23^{\circ}$.

the dSpace/Control Desk software program. Although it introduced some inertial forces, the pantographic arm enabled us to reliably and reproducibly test the performances of the BeeRotor robot under safe flying conditions, while making the parameter monitoring simpler.

\section{BEEROTOR'S MINIATURE QUASI-PANORAMIC EYE AND ITS OPTIC FLOW PROCESSING}

The BeeRotor's eye is composed of four 2-gram stand-alone visual motion sensors (see Fig. $3 c$ and 4a) looking UpForward (UF), Up-Backward (UB), Down-Forward (DF) and Down-Backward (DB), as shown in Figure 3d. Each visual motion sensor is tilted from the vertical axis at an angle of $23^{\circ}$ and has a longitudinal Field-Of-View (FOV) of $24^{\circ}$. Each of the 4 visual motion sensors used on the BeeRotor robot is based on a 6-pixel off-the-shelf photosensor array (iC-LSC from iCHaus Company, http://www.ichaus.de) mounted on a lightweight lens (Sparkfun SEN-00637, focal length 2mm, f-number 2.8) borrowed from a mobile telephone camera lens. An on-chip current amplifier is integrated into each photodiode, but a programmble gain was recently added to the circuit so as to be able to use the maximum range of the Analog to Digital Converter (see Fig. 4b) [26]. The optic flow is computed using the "time of travel" scheme (see Fig. 4b) which assesses the angular speed $\omega_{i}$ of any contrasting object detected by two neighboring photosensor signals by measuring the time lag:

$$
\Delta t_{i}=\frac{\triangle \varphi}{\omega_{i}}
$$

where $\Delta \varphi$ is the inter-receptor angle (the angle between two adjacent optical axes). Like the Angular Sensitivity Function (ASF) of a single fly photoreceptor, each photosensor features a Gaussian angular sensitivity function [39], which can be adjusted by defocusing the lens from the sensor so that an appropriate bell-shaped ASF is projected onto the photosensor array, as occurs in some diurnal insects [40] with:

$$
\Delta \varphi=\Delta \rho=4^{o}
$$

where $\Delta \rho$ is the acceptance angle. This defocusing acts as 

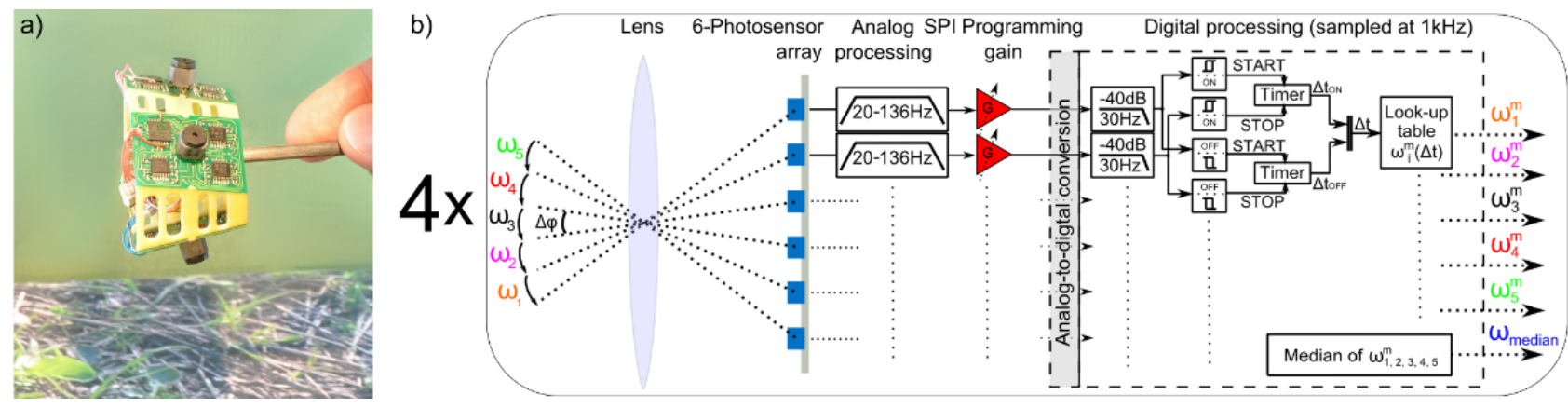

Fig. 4. a) BeeRotor's eye with its four electronic boards composed of 5 adjacent programmable-gain visual motion sensors called "time of travel" EMDs. b) Processing architecture of one visual motion sensor. The visual signals emitted by each photoreceptor are filtered through an analog bandpass filter and a second order fixed-point digital low-pass filter. Before being digitized, the visual signals are amplified using the programmable gains controlled by a SPI bus in order to finely tune the dynamic range of the signals with respect to the ambient illuminance. Two neighboring signals are then thresholded. In parallel, the "time of travel" $\Delta t$ elapsing between the two signals is measured by a timer: $\Delta t_{O N}$ and $\Delta t_{O F F}$ are measured, based on ON and OFF contrast distinguishing processes. These $\Delta t$ are used to generate the 1-D angular speed $\omega_{i}^{m}$ within the visual field of each 1-D local motion sensor. Lastly, the 5 local motion sensors are combined to generate a robust and frequently refreshed 1-D median angular speed.

a spatial low-pass filtering process on the visual signals, which are then temporally filtered and amplified by means of the programmable gains which make it possible to finely tune the dynamic range of the visual signals with respect to the ambient illuminance. The other processing steps have been described in detail in [25]. Two separate processing pathways distinguish between ON (dark to light) and OFF (light to dark) contrasts, as previously found to occur in flying insects [20], [41]. Our visual motion sensor generates 5 simultaneous local measurements of the 1-D angular speed generated by a natural scene within a measurement range of more than one decade $\left[30^{\circ} / s ; 350^{\circ} / s\right]$. It also computes the median value $\omega_{\text {median }}$ of the 5 local motion sensors. The whole processing of the 5 local motion sensors and the calculation of the median value are carried out on a dsPIC33FJ128GP802 microcontroller working at a sampling frequency of $1 \mathrm{kHz}$. The microcontroller embedded onboard each visual motion sensor is connected to the main electronic board via a SPI bus.

\section{BEEROTOR VISUOMOTOR CONTROL LOOPS}

The autopilot embedded in the BeeRorot robot is composed of two intertwined feedback loops controlling the pitch angle $\theta$ and the thrust (Fig. 5). The optic flow generated by the aircraft moving through the experimental environment was measured by the 4 visual motion sensors. The median value measured by each visual motion sensor $\left(\omega_{\text {medianUF }}, \omega_{\text {medianU } B}, \omega_{\text {medianDF }}, \omega_{\text {medianDB }}\right)$ was used to compute the ventral and dorsal optic flow, called $\omega_{V t r l}$ and $\omega_{D r s l}$ :

$$
\begin{aligned}
& \omega_{V t r l}=\frac{1}{\cos ^{2}(\alpha)}\left(\omega_{\text {median } D F}+\omega_{\text {median } D B}\right) \\
& \omega_{D r s l}=\frac{1}{\cos ^{2}(\alpha)}\left(\omega_{\text {medianU } F}+\omega_{\text {medianUB }}\right)
\end{aligned}
$$

Each visual motion sensor is tilted around the robot's pitch axis at an angle of $\alpha= \pm 23^{\circ}$. The sum $\omega_{V t r l}+\omega_{D r s l}$ is then compared with the Forward Speed OF set-point $\omega_{\text {setFwdSpeed }}$ in order to adjust the robot's pitch angle $\theta$, which eventually determines the groundspeed $V_{x}$. When the sum $\omega_{V t r l}+\omega_{D r s l}$ is greater than the Forward Speed OF set-point $\omega_{\text {setFwdSpeed }}$, the surge controller will decrease the robot's pitch angle $\theta$ in order to slow down the rotorcraft.

The nearest wall is determined thanks to the maximum value reached between $\omega_{V t r l}$ and $\omega_{D r s l}$ and this value is compared with the Altitude OF set-point $\omega_{\text {set Alt }}$ and used to control the rotors' thrust, which will eventually determine the altitude $\mathrm{z}$. When the OF of the nearest wall is greater than the Altitude OF set-point $\omega_{\text {set Alt }}$, depending on which wall is detected, the heave controller will either increase or decrease the rotor speed command in order to drive the rotorcraft away from this wall.

During these experiments, the angle of the eye with respect to the body was dynamically controlled to compensate for the pitch angle $\theta$ of the aircraft, so that the eye would be always oriented vertically "upward". The illuminance, reflected by the ground and the ceiling scenes and provided by the natural light coming through the windows, was around 200lux.

\section{Altitude And Speed control based on Dorsal AND VENTRAL OF REGULATION}

\section{A. Surface following}

Figures 6 and 7 show the performances of the BeeRotor robot while automatically following the ceiling and the ground, respectively. In both cases, the feedback loops of the robot were activated after covering a distance of $6 \mathrm{~m}$, when the aircraft was already flying at a constant speed and altitude. When the regulation process was activated, the altitude $\mathrm{z}$ and the horizontal speed $V_{x}$ of the aircraft changed in response to the two intertwined feedback loops acting respectively on the pitch angle $\theta$ and the rotors' thrust of the robot which mainly drives the lift and therefore the altitude as the pitch 


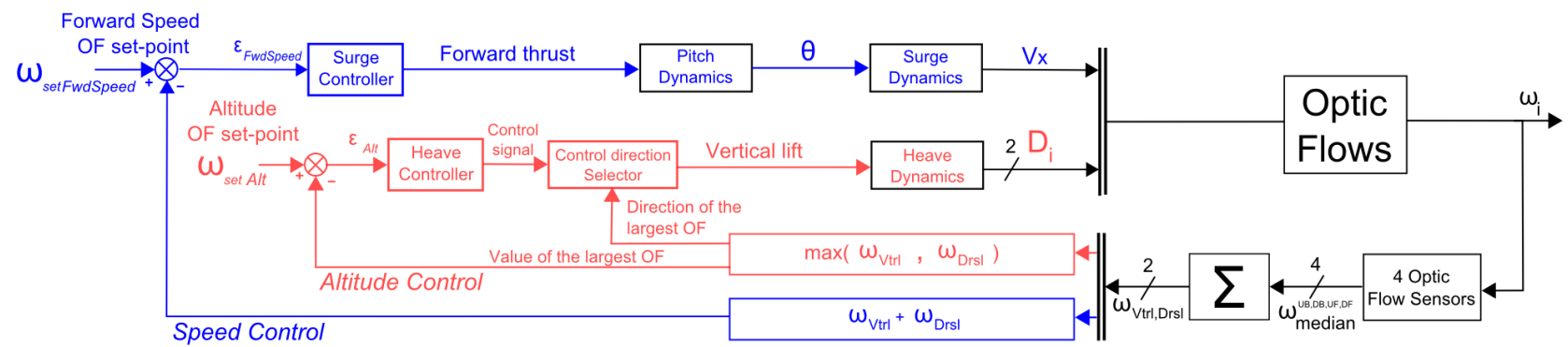

Fig. 5. The BeeRotor robot is regulated by two intertwined feedback loops acting on the pitch angle $\theta$ and the thrust. First, the sum of the 2 median optic flows generated by the ground and the ceiling is compared with the Forward Speed OF set-point $\omega_{\text {setFwdSpeed }}$ to control the pitch angle $\theta$ of the robot, which will eventually determine the groundspeed $V_{x}$ (blue feedback loop). Secondly, the optic flow of the nearest wall is compared with the Altitude OF set-point $\omega_{\text {set } A l t}$ in order to adjust the thrust, which will eventually determine the altitude $\mathrm{z}$ (red feedback loop).
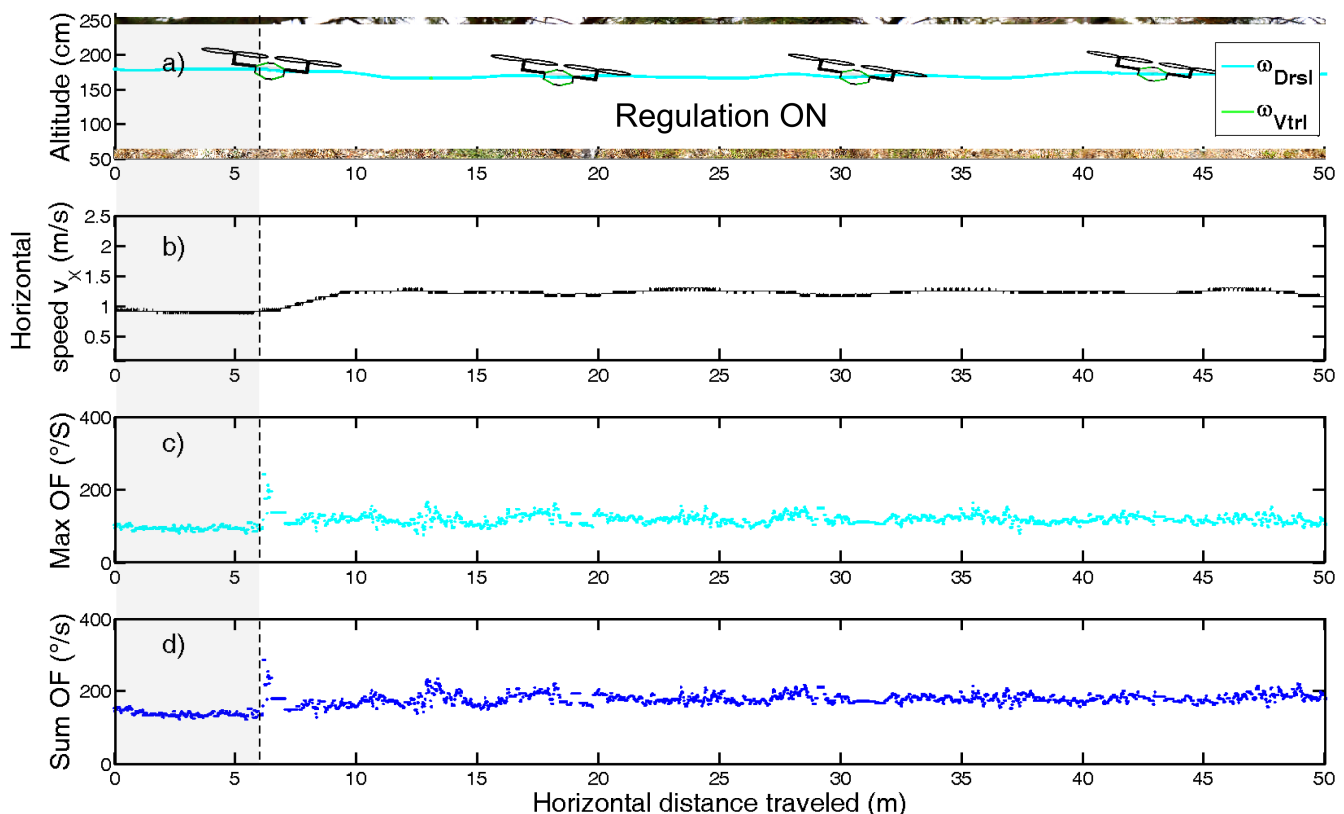

Fig. 6. Automatic ceiling following controlled by the optic flow. The regulation process was activated after the robot had covered a distance of $6 \mathrm{~m}$. a) Altitude of the BeeRotor robot in the 12-m long naturally contrasted environment when it was following the ceiling. The altitude is plotted in cyan when the nearest wall detected was the ceiling and in green when the nearest wall detected was the ground. The regulation process was activated after covering a distance of $6 \mathrm{~m}$ when the nearest wall detected was the ceiling. The BeeRotor robot therefore adjusted its altitude depending on the Altitude OF set-point $\omega_{\text {set }}$ Alt while following the ceiling. b) Horizontal speed of the aircraft while following the ceiling. When the regulation process was activated, the horizontal speed $V_{x}$ of the rotorcraft increased due to the speed feedback loop. c) OF of the nearest wall. When the regulation system was ON, the robot regulated its thrust so as to stay around its Altitude OF set-point $\omega_{s e t A l t}$. d) Sum $\omega_{V t r l}+\omega_{D r s l}$. When the regulation system was ON, the aircraft regulated its pitch angle $\theta$ so as to maintain the sum $\omega_{V t r l}+\omega_{D r s l}$ around its Forward Speed OF set-point $\omega_{\text {setFwdSpeed }}$.

angle never exceeded $20 \mathrm{deg}$. After travelling a few meters, the rotorcraft stabilized at a fixed altitude and horizontal speed, depending only on the Altitude OF set-point $\omega_{\text {set Alt }}$ and the Forward Speed OF set-point $\omega_{\text {setFwdSpeed }}$. As the two set-points were the same in both experiments, the robot's steady-state horizontal speed $V_{x}$ was the same in both cases although the initial speed was different. However, the surface followed was not the same in both experiments because the aircraft's initial altitude was different: the fact that the rotorcraft was initially nearer the ceiling, in the first experiment, generated ceiling following behavior, whereas the fact that it was initially nearer the ground in the second experiment generated a ground following response.

The influence of the aircraft's initial altitude can be seen in figure 8. In these six experiments, the regulation process was activated after the robot had covered a distance of $6 \mathrm{~m}$, starting at an altitude ranging between 80 and $220 \mathrm{~cm}$. The altitude is plotted in cyan when the nearest wall detected was the ceiling and in green, when the nearest wall detected was the ground. As was to be expected, depending on the nearest wall detected when the regulation process was activated, the BeeRotor robot could follow either the ground or the ceiling. After a short time, the aircraft reached its final altitude, which was similar in all the experiments where the robot was following the ceiling and in all those where the robot was following the ground. In any case, the rotorcraft quickly reached a safe distance from the nearest wall regardless of the initial altitude. In one experiment starting at an altitude 

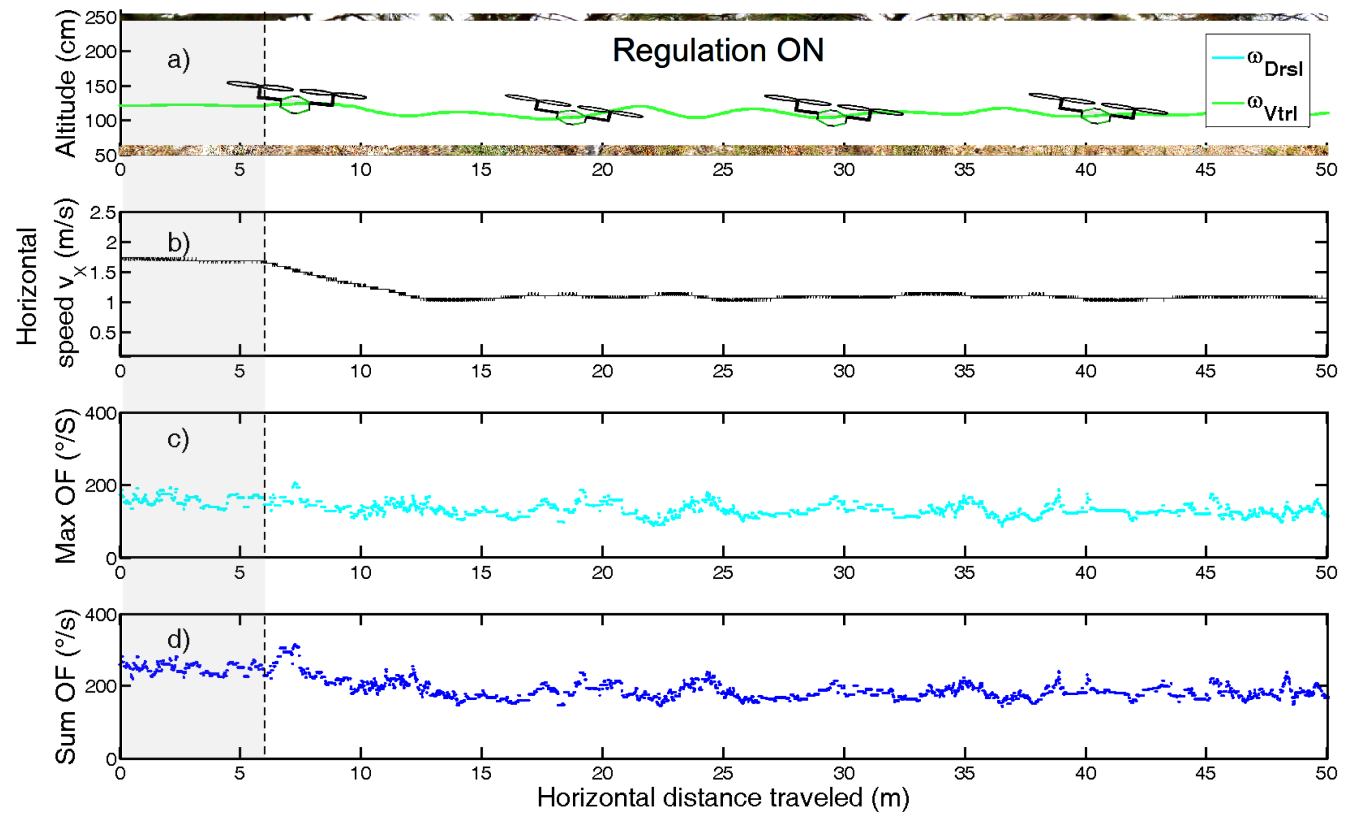

Fig. 7. Automatic ground following controlled by the optic flow. The regulation process was activated after covering a distance of $6 \mathrm{~m}$. a) Altitude of the BeeRotor robot when following the ground in the 12-m long naturally contrasted experimental environment. The altitude is plotted in cyan when the nearest wall detected was the ceiling and in green, when the nearest wall detected was the ground. The regulation process was activated after covering a distance of $6 \mathrm{~m}$ when the nearest wall detected was the ground. The BeeRotor robot therefore adjusted its altitude, depending on the Altitude OF set-point $\omega_{\text {set }} A l t$ while following the ground. b) Horizontal speed of the aircraft while following the ground. When the regulation system was activated, the horizontal speed $V_{x}$ of the rotorcraft decreased due to the speed feedback loop. c) OF of the nearest wall. When the regulation was ON, the robot regulated its thrust so as to stay around its Altitude OF set-point $\omega_{\text {set Alt }}$. d) Sum $\omega_{V t r l}+\omega_{D r s l}$. When the regulation was ON, the aircraft regulated its pitch angle $\theta$ so as to maintain the sum $\omega_{V t r l}+\omega_{D r s l}$ around its Forward Speed OF set-point $\omega_{\text {setFwdSpeed }}$.

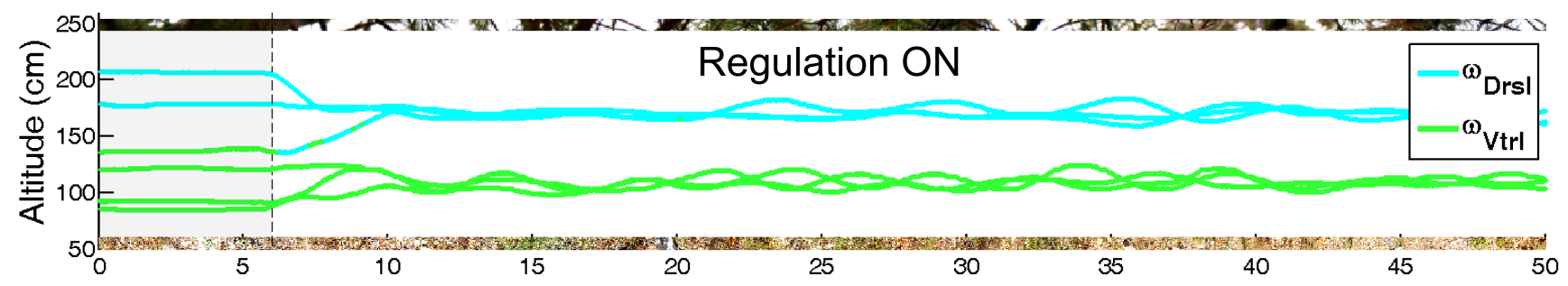

Fig. 8. Automatic ground and ceiling surface following, starting at various altitudes. In these six experiments, the regulation process was activated after the aircraft had covered a distance of $6 \mathrm{~m}$, starting at an altitude ranging between 80 and $220 \mathrm{~cm}$. Depending on the nearest wall detected when the regulation system was activated, the BeeRotor robot followed the ground as well as the ceiling. In any case, the rotorcraft quickly reached a safe distance from the nearest wall, regardless of the initial altitude.

which was very near the middle of the range, the BeeRotor robot followed the ceiling, whereas the nearest wall detected before the regulation process was activated was the ground, because shortly after the regulation process was triggered, the aircraft took the ceiling to be the nearest wall, and therefore adopted ceiling following behavior.

\section{B. Automatic vertical docking on the ceiling}

To induce automatic docking, the Forward Speed OF setpoint $\omega_{\text {setFwdSpeed }}$ was gradually decreased so as to gradually decrease the horizontal speed $V_{x}$. In order to keep the maximum optic flow constant, the altitude control loop gradually decreased the distance between the nearest wall detected and the aircraft, so that the latter eventually docked with a negligible forward speed at touchdown. Fig. 9a shows the docking trajectory observed when the Forward Speed OF set-point $\omega_{\text {setFwdSpeed }}$ was reduced (see Fig. 9c) rampwise from $400^{\circ} / s$ to $20^{\circ} / s$ (in a $50^{\circ} / s$ ramp). The decrease in the Forward Speed OF set-point $\omega_{\text {setF }}$ wdSpeed decreased the horizontal speed $V_{x}$, as can bee seen in Figure 9b. The Altitude control loop gradually increased the altitude of the aircraft, bringing it nearer to the ceiling, and thus maintaining the maximum optic flow around the constant Altitude OF set-point $\omega_{\text {set } A l t}$.

\section{CONCLUSION}

In the present experiments performed indoors with a new miniature $(80 \mathrm{~g})$ tandem rotorcraft called BeeRotor, reputedly difficult manoeuvers of the kind that will have to be performed safely by the miniature unmanned aerial vehicles of the future, such as ground and ceiling following and auto- 

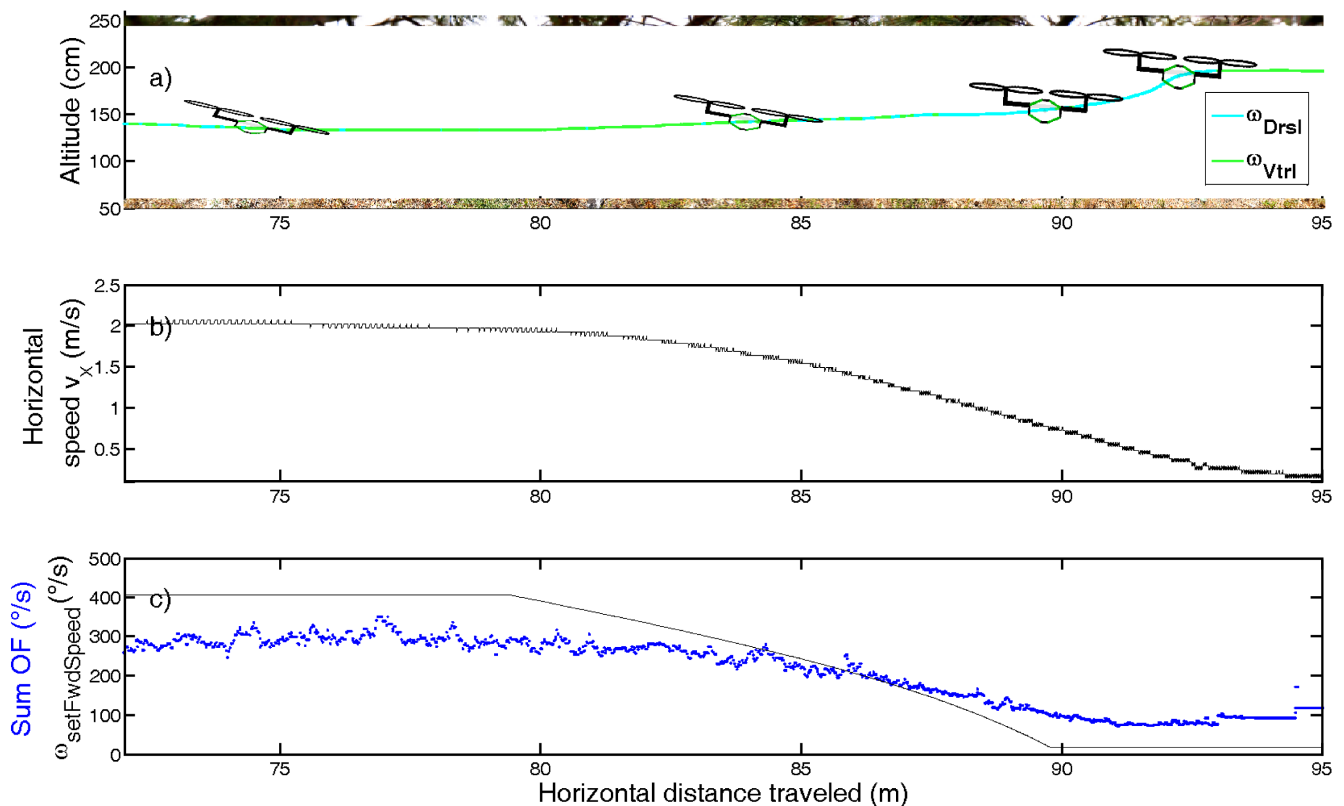

Fig. 9. Automatic docking of the aircraft on the ceiling achieved by dynamically changing the Forward Speed OF set-point $\omega_{\text {set } F w d S p e e d}$. a) Altitude of the BeeRotor robot in the 12-m long naturally contrasted environment during automatic docking. The altitude is plotted in cyan when the nearest wall detected is the ceiling and in green when the nearest wall detected is the ground. Automatic docking is induced by decreasing the Forward Speed OF set-point $\omega_{\text {setFwdSpeed }}$, which decreases the horizontal speed $V_{x}$ while keeping the Altitude OF set-point $\omega_{\text {set Alt }}$ constant. The BeeRotor robot therefore increases its altitude to compensate for the decrease in the optic flow due to the deceleration and moves closer to the ceiling, which results in automatic docking at a horizontal speed of almost zero. b) Horizontal speed of the BeeRotor robot during automatic docking. As was to be expected, by dynamically decreasing the Forward Speed OF set-point $\omega_{\text {setF } w d S p e e d}$, the aircraft gradually reduced its horizontal speed to almost zero, resulting in a safe docking. c) Sum of the ventral and dorsal optic flows $\omega_{V t r l}+\omega_{D r s l}$ in blue and the Forward Speed OF set-point $\omega_{\text {set FwdSpeed }}$ in black. The automatic docking was induced by generating a ramp on the Forward Speed OF set-point $\omega_{\text {setFwdSpeed }}$ from $400^{\circ} / s$ to $20^{\circ} / s$.

matic docking, were carried out successfully. The autopilot mounted onboard this robot uses only optic flow sensors to regulate the ventral and dorsal optic flow by adjusting the thrust and the pitch angle of the aircraft via two visuomotor control loops. By regulating the maximum optic flow generated by the ground or the ceiling and the sum of these optic flows, it is now possible for a robot of this kind to automatically reach a "safe height and forward speed" regardless of the starting altitude and forward speed without being provided with any information about the height, the distance to the surrounding walls or the airspeed, which are usually measured onboard conventional aircraft. The BeeRotor robot also proved to be able to dock automatically by gradually decreasing the Forward Speed OF set-point, which causes the aircraft to decelerate and therefore to automatically fly closer to one of the surrounding walls, thus maintaining a "safe height" that suitably matches its speed $V_{x}$ by keeping the ratio between these two variables constant. In the case it would have followed the ground, the aircraft would therefore have gradually reduced the distance from the ground based on OF measurements alone and landed automatically, and would not require a ground control station to be able to land with a negligible forward speed at touchdown. The autonomous BeeRotor aircraft inspired by the insect world, especially in terms of its visual processing system, which was based on the results of electrophysiological experiments on living insects, is capable of producing great performances although it requires remarkably few resources. Biologically inspired robots equipped with minimum computational resources and a very light payload can therefore provide MAVs with reliable alternative means of performing complex flying manoeuvers.

In the near future, the servomotor controlling the pitch angle of the BeeRotor robot will be removed and the forward speed of the robot will be controlled via the differential speed of the two propellers.

Since automatic docking, surface following and collision avoidance are still challenging issues for designing Micro Aerial Vehicles for use in natural environments, the BeeRotor robot is a promising proof of concept, which constitutes a further step toward producing fully autonomous microflyers capable of navigating in an unknown environment with an extremely light payload. Robots of this kind could be used in several fields of application, such as search and rescue missions, reconnaissance and planetary landings, contexts where sensors such as GPS devices cannot be used.

\section{ACKNOWLEDGMENT}

We are very grateful to $\mathrm{M}$. Boyron for his involvement in the electronic design, F. Paganucci, D. Dray, Y. Luparini and J. Diperi for their help with the mechanical design, F. Roubieu for his kind help with the photographs, J. Blanc for improving the English manuscript and G. Portelli, J. Serres, N. Franceschini and S. Viollet for their fruitful comments and suggestions during this research. 


\section{REFERENCES}

[1] N. Franceschini, J. M. Pichon, and C. Blanes, "From insect vision to robot vision," Philosophical Transactions of the Royal Society B: Biological Sciences, vol. 337, pp. 283-294, 1992.

[2] W. Green, P. Oh, K. Sevcik, and G. Barrows, "Autonomous landing for indoor flying robots using optic flow," in ASME International Mechanical Engineering Congress (IMECE), Washington, D.C., USA, November 2003.

[3] F. Ruffier and N. Franceschini, "Visually guided micro-aerial vehicle: automatic take off, terrain following, landing and wind reaction," in Proceeding of IEEE International Conference on Robotics and Automation (ICRA), New Orleans, USA, 2004, pp. 2339-2346.

[4] W. Green, P. Oh, and G. Barrows, "Flying insect inspired vision for autonomous aerial robot maneuvers in near-earth environments," in IEEE International Conference on Robotics and Automation (ICRA), vol. 3, New Orleans, USA, 2004, pp. 2347 - 2352.

[5] J.-C. Zufferey and D. Floreano, "Fly-inspired visual steering of ultralight indoor aircraft," IEEE Transactions on Robotics, vol. 22(1), pp. 137-146, 2006.

[6] M. Garratt and J. Chahl, "Vision-based terrain following for an unmanned rotorcraft," Journal of Field Robotics, vol. 25, pp. 284 301, 2008.

[7] A. Beyeler, J.-C. Zufferey, and D. Floreano, "Optipilot: control of takeoff and landing using optic flow," in European Micro Aerial Vehicle Conference (EMAV), Delft, Netherlands, 2009, pp. 1-8.

[8] F. G. Bermudez and R. Fearing, "Optical flow on a flapping wing robot," in IEEE International Conference on Intelligent Robots and Systems (IROS), St. Louis, USA, 2009, pp. 5027-5032.

[9] J. Conroy, G. Gremillion, B. Ranganathan, and J. Humbert, "Implementation of wide-field integration of optic flow for autonomous quadrotor navigation," Autonomous robots, vol. 27 (3), pp. 189-198, 2009.

[10] B. Herisse, T. Hamel, R. Mahony, and F.-X. Russotto, "The landing problem of a VTOL unmanned aerial vehicle on a moving platform using optical flow," in IEEE International Conference on Intelligent Robots and Systems (IROS), Taipei, Taiwan, 2010, pp. 77 - 89.

[11] S. Zingg, D. Scaramuzza, S. Weiss, and R. Siegwart, "MAV navigation through indoor corridors using optical flow," in IEEE International Conference on Robotics and Automation (ICRA), Anchorage, USA, May 2010, pp. $3361-3368$.

[12] G. de Croon, E. de Weerdt, C. De Wagter, B. Remes, and R. Ruijsink, "The appearance variation cue for obstacle avoidance," in IEEE International Conference on Robotics and Biomimetics (ROBIO), Tianjin, China, 2010, pp. 1606 - 1611.

[13] S. Fuller and R. Murray, "A hovercraft robot that uses insect-inspired visual autocorrelation for motion control in a corridor," in IEEE Conference on Robotics and Biomimetics (ROBIO), Phucket, Thailand, 2011, pp. 1474-1482.

[14] G. Barrows and C. Neely, "Mixed-mode VLSI optic flow sensors for in-flight control of a Micro Air Vehicle," in SPIE : Critical technologies for the future of computing, vol. 4109, San Diego, CA, USA, Aug 2000, pp. 52-63.

[15] J. Santos-Victor, G. Sandini, F. Curotto, and S. Garibaldi, "Divergent stereo in autonomous navigation: From bees to robots," International Journal of Computer Vision, vol. 14, pp. 159-177, 1995.

[16] S. Griffiths, J. Saunders, A. Curtis, B. Barber, T. McLain, and R. Beard, "Maximizing miniature aerial vehicles," Robotics \& Automation Magazine, vol. 13, pp. 34-43, 2006.

[17] A. Beyeler, J.-C. Zufferey, and D. Floreano, "Vision-based control of near-obstacle flight," Autonomous robots, vol. 27, pp. 201-219, 2009.

[18] H. Dahmen, A. Millers, and H. A. Mallot, "Insect inspired odometry by optic flow recorded with optical mouse chips," in Flying insects and robots, D. Floreano, J.-C. Zufferey, M. V. Srinivasan, and C. E. Ellington, Eds. Springer, Berlin, Eds : Floreano, D. and Zufferey,J. C. and Srinivasan, M. V. and Ellington, C., 2009, pp. 115-126.

[19] C. Blanes, Appareil visuel elementaire pour la navigation a vue d'un robot mobile autonome. Marseille: Master thesis in Neurosciences (DEA in French), advisor N. Franceschini, Neurosciences, Univ. AixMarseille II, 1986.

[20] N. Franceschini, A. Riehl, and A. le Nestour, "Directionally selective motion detection by insect neurons," in Facets of Vision, D. Stavenga and R. Hardie, Eds. Berlin \& New York, Springer, 1989, pp. 360-390.
[21] S. Viollet and N. Franceschini, "Super-accurate visual control of an aerial minirobot," In Autonomous minirobots for research and edutainment (AMIRE), vol. 1, pp. 215-224, 2001.

[22] F. Ruffier and N. Franceschini, "Optic flow regulation: the key to aircraft automatic guidance," Robotics and Autonomous Systems, vol. 50, no. 4, pp. 177 - 194, 2005.

[23] S. Viollet, F. Ruffier, T. Ray, M. Menouni, F. Aubépart, L. Kerhuel, and N. Franceschini, "Characteristics of three miniature bio-inspired optic flow sensors in natural environments," in SENSORCOMM, Venice, Italy, 2010, pp. $51-55$.

[24] F. Expert, S. Viollet, and F. Ruffier, "Outdoor field performances of insect-based visual motion sensors," Journal of Field Robotics, vol. 28 (4), pp. 529-541, 2011.

[25] F. Roubieu, F. Expert, M. Boyron, B.-J. Fuschlock, S. Viollet, and F. Ruffier, "A novel 1-gram insect based device measuring visual motion along 5 optical directions," in IEEE Sensors 2011 conference, Limerick, Ireland, 2011, pp. 687-690.

[26] F. Ruffier and F. Expert, "Visual motion sensing onboard a 50-g helicopter flying under complex vicon-lighting conditions," in IEEE International Conference on Complex Medical Engineering (CME), Kobe, Japan, July 2012, pp. 634-639.

[27] F. Expert, S. Viollet, and F. Ruffier, "A mouse sensor and a 2-pixel motion sensor exposed to continuous illuminance changes," in IEEE Sensors 2011 conference, Limerick, Ireland, 2011, pp. 974-977.

[28] F. Ruffier and N. Franceschini, "Octave, a bioinspired visuo-motor control system for the guidance of micro-air vehicles," in Bioengineered and Bioinspired Systems, A. Rodriguez-Vazquez, D. Abbott, R. Carmona, Eds. Bellingham, U.S.A: SPIE Vol. 5119, 2003, pp. 1-12.

[29] N. Franceschini, F. Ruffier, and J. Serres, "A bio-inspired flying robot sheds light on insect piloting abilities," Current Biology, vol. 17, pp. 329 - 335, 2007.

[30] J. Serres, G. Masson, F. Ruffier, and N. Franceschini, "A bee in the corridor: centering and wall-following," Naturwissenschaften, vol. 95, pp. 1181-1187, 2008.

[31] G. Portelli, J. Serres, F. Ruffier, and N. Franceschini, "Modelling honeybee visual guidance in a 3-d environment," Journal of PhysiologyParis, vol. 104, pp. 27-39, 2010.

[32] J. Serres, D. Dray, F. Ruffier, and N. Franceschini, "A vision-based autopilot for a miniature air vehicle: joint speed control and lateral obstacle avoidance," Autonomous robots, vol. 25, no. 1, pp. 103-122, 2008

[33] G. Portelli, F. Ruffier, and N. Franceschini, "Honeybees change their height to restore their optic flow," Journal of Comparative Physiology, vol. 196 (4), pp. 307-313, 2010.

[34] G. Portelli, F. Ruffier, F. Roubieu, and N. Franceschini, "Honeybees' speed depends on dorsal as well as lateral, ventral and frontal optic flows," PLOS ONE, vol. 6 (5), p. doi:10.1371/journal.pone.0019486, 2011.

[35] M. Srinivasan, S. Zhang, J. Chahl, E. Barth, and S. Venkatesh, "How honeybees make grazing landings on flat surfaces," Biological Cybernetics, vol. 83, pp. 171-183, 2000.

[36] J. Santos-Victor and G. Sandini, "Visual behaviors for docking," Computer Vision and Image Understanding, vol. 67(3), pp. 223-238, 1997.

[37] C. McCarthy and N. Barnes, "A unified strategy for landing and docking using spherical flow divergence," in IEEE Transactions on Pattern Analysis and Machine Intelligence, vol. (T-PAMI), 2012, p. accepted.

[38] J.-C. Zufferey, A. Beyeler, and D. Floreano, "Autonomous flight at low altitude using light sensors and little computational power," Journal of Micro Air Vehicles, vol. 2 (2), pp. 107-117, 2010.

[39] K. Götz, "Optomotorische Untersuchung des visuellen systems einiger Augenmutanten der Fruchtfliege Drosophila," Biological Cybernetics, vol. 2, pp. 77-92, 1964.

[40] M. Land, "Visual acuity in insects," Annual Review of Entomology, vol. 42, pp. 147-17, 1997.

[41] H. Eichner, M. Joesch, B. Schnell, D. Reiff, and A. Borst, "Internal structure of the fly elementary motion detector," Neuron, vol. 70, pp. 1155-1164, 2011. 